\title{
ESTUDO DA QUANTIFICAÇÃO DE LIPÍDIOS MICROALGAIS ATRAVÉS DE FLUORESCÊNCIA 2D
}

\author{
L. BRESOLIN ${ }^{1}$, C. RANZAN ${ }^{1}$, N. N. RAMIREZ1, M. FARENZENA ${ }^{1}$, L. F. \\ TRIERWEILER ${ }^{1}$, J. O. TRIERWEILER ${ }^{1}$ \\ ${ }^{1}$ Grupo de Intensificação, Modelagem, Simulação, Controle e Otimização de Processos \\ (GIMSCOP) \\ Departamento de Engenharia Química, UniversidadeFederal do Rio Grande do Sul (UFRGS) \\ E-mail para contato: \{larisaab, cassiano, farenz, luciane, jorge $@ @ e n q . u f r g s . b r$
}

RESUMO - A crescente necessidade por combustíveis alternativos faz com que sejam necessárias pesquisas sobre novas fontes energéticas eficientes e não agressivas ao meio ambiente. Neste cenário, as microalgas têm se apresentado como uma alternativa para a produção de biodiesel. Em virtude disso, torna-se imprescindível a utilização de um método de quantificação lipídica microalgal que seja rápido e confiável. Visando essa necessidade, estudou-se o desenvolvimento de uma metodologia de quantificação lipídica baseada na técnica de Espectroscopia Fluorescente 2D. Modelos quimiométricos foram propostos e calibrados usando soluções de azeite em acetona, medidas de EF2D e seleção multivariável PSCM. Modelos multilineares de oito pares de fluorescência apresentaram os melhores resultados na caracterização de lipídios, passando para teste em cultivos reais de microalgas em diferentes porcentagens de vinhaça ( $0 \%$, $8,1 \%, 20 \%$ e $31,9 \%$ ). A aplicação direta do método não apresentou resultados quantitativos aceitáveis (menor erro de medida na ordem de 7\%), entretanto a tendência foi capturada de forma satisfatória, indicando a viabilidade da técnica proposta.

\section{INTRODUÇÃO}

A cada ano o consumo de derivados do petróleo, como gasolina e diesel, e do álcool proveniente da cana de açúcar ou milho, tende a subir, visto o aumento da frota de veículos e da expansão econômica que impulsiona a produtividade do setor industrial. Portanto, torna-se imprescindível a busca de fontes energéticas renováveis que sejam menos agressivas ao meio ambiente e que, juntamente, apresentem rentabilidade econômica. 
Um combustível que se apresenta como alternativa aos combustíveis derivados do petróleo é o biodiesel, que pode ser usado em qualquer motor a diesel sem a necessidade de modificações neste, proveniente de fontes renováveis como soja, girassol ou outras oleaginosas. No entanto, utilizar essas sementes para a produção de biodiesel acarreta uma perda para o setor alimentício, uma vez que a produção não é mais destinada à alimentação e o espaço físico de plantio também fica comprometido. Biodiesel a partir de sementes oleaginosas e bioetanol proveniente da cana-de-açúcar e milho vêm sendo produzido em quantidades crescentes como biocombustíveis de fontes renováveis, mas sua produção em grandes quantidades não é sustentável. Com o objetivo de sanar estas lacunas de produção, nos últimos anos vem sendo estudada como alternativa de grande destaque a produção de biodiesel a partir de microalgas (Chisti 2008).

As microalgas têm sido reconhecidas como uma produtora de matéria-prima para a produção de biodiesel por causa de sua elevada eficiência fotossintética, rápida taxa de crescimento e habilidade de acumular significativas quantidades de lipídios (Govender, Ramanna et al. 2012). O uso de microalgas envolve vários aspectos vantajosos para o aproveitamento energético de sua biomassa, tais como: apresentam um curto ciclo de vida e uma alta taxa de crescimento; permitem diversas condições de cultivo, além de possibilitarem produção o ano inteiro; não há necessidade do uso de agrotóxicos; requerem uma área reduzida para o cultivo e, consequentemente, utilizam um reduzido volume de água potável.

Quanto maior a quantidade de lipídios provenientes da produção de microalgas, maior a capacidade de produção de biodiesel, por isso, além de diversos estudos analisarem diferentes espécies de microalgas, também é de grande necessidade a capacidade de quantificação desses lipídios. Esta é usualmente realizada por meios gravimétricos após extração com solvente, o que requer um tempo e gasto consideráveis, além de grande quantidade de biomassa seca (Govender, Ramanna et al. 2012).

Um método de detecção de lipídios de microalgas que tem se mostrado promissor e acessível é através de técnicas de marcação empregando espectrofluorescência (Govender, Ramanna et al. 2012). Essa técnica consiste basicamente em adicionar determinada solução de corante na amostra retirada do cultivo de microalga, onde a mistura fica em contato durante o tempo adequado para que o corante atravesse a parede celular da microalga, conseguindo assim marcar os lipídios que, então, poderão ser analisados através da fluorescência resultante desta interação corante/lipídio. Os corantes comercialmente disponíveis que possuem essa característica são o Vermelho do Nilo e o BODIPY 505/515.

A análise de dados de espectroscopia fluorescente 2D é feita através de métodos quimiométricos, como PCA (Principal Component Analysis), PCR (Principal Component Regression), PLSR (Partial Least Squares Regression), PSCM (Pure Spectral Component Modeling), entre outros, que consistem em metodologias capazes de extrair informações relacionadas às variáveis de estado, nesse caso concentração lipídica, contidas em grandes matrizes de dados de espectroscopia (Clementschitsch, Jürgen et al. 2005). 


\section{MATERIAIS E MÉTODOS}

\subsection{Quantificação de Lipídios Microalgais}

A metodologia de análise empregada neste trabalho baseia-se na comparação entre variações da matriz espectral em função de variações na variável de interesse (concentração de lipídio). Desta forma, ocorre a necessidade de amostras previamente caracterizadas para a calibração do método analítico proposto para, então, posteriormente caracterizar as amostras de interesse.

Azeite de oliva foi escolhido como sendo um possível representante dos lipídios produzidos pelas microalgas em virtude de sua fácil obtenção e perfil de ácidos graxos próximo ao perfil de ácidos graxos da microalga Scenedesmus sp.. A calibração do método analítico é baseada na caracterização de soluções de azeite em acetona. A acetona foi definida como solvente para o azeite em virtude da solução de Vermelho do Nilo ser feita com a mesma, evitando assim que novos solventes fossem introduzidos na matriz espectral.

Para a avaliação do método analítico de quantificação de lipídios foram feitas soluções com diferentes concentrações de azeite de oliva, marca Oikos (Grécia), em acetona ( $0 \%, 1 \%$, $5 \%, 10 \%, 20 \%, 30 \%, 40 \%$ e $50 \%$ em volume). Para a solução de análise foram utilizadas alíquotas de $50 \mu \mathrm{L}$ das soluções padrão de azeite, $150 \mu \mathrm{L}$ da solução de Vermelho no Nilo (10 $\mu \mathrm{g} / \mathrm{mL}$ em acetona) e $2,8 \mathrm{~mL}$ de acetona, totalizando $3 \mathrm{~mL}$. A concentração final de Vermelho do Nilo foi de $0,5 \mu \mathrm{g} / \mathrm{mL}$, conforme recomendado por Chen et al. (2009).

\subsection{Cultivo de Microalgas}

Meio de cultivo: O meio Guillard Modificado foi usado para o crescimento de cultivo puro da Scenedesmus sp.. Maiores informações da preparação e composição do meio Guillhard Modificado são encontradas no trabalho de Ramirez (2013).

Fotobiorreatores: Os reatores usados neste experimento são do tipo fotobiorreatores airlift, feitos de acrílico com dimensões de $35 \mathrm{~cm}$ de altura, $17 \mathrm{~cm}$ de largura e $6 \mathrm{~cm}$ de profundidade com placa central de $27 \mathrm{~cm}$ x $17 \mathrm{~cm}$. O volume de cultivo é de aproximadamente 3 litros. A planta usada para realizar os experimentos tem capacidade para a instalação de doze fotobiorreatores e permite o controle de vazão de $\mathrm{CO} 2$, intensidade luminosa, temperatura e fotoperíodo (descrições mais detalhadas da planta de fotobiorreatores podem ser obtidas no trabalho de Gris (2013)).

Cultivo de Microalgas: Os cultivos da microalga Scenedesmus sp. foram feitos em duplicata em quatro diferentes condições, cultivo com $0 \%$ de vinhaça, 8,1\% de vinhaça, 20\% de vinhaça e 31,9\% de vinhaça. Maiores detalhes sobre a metodologia de cultivo e composição da podem ser encontrados no trabalho de Ramirez (2013). A escolha por realizar os cultivos com diferentes porcentagens de vinhaça foi baseada no trabalho de Ramirez (2013), no qual foi discutida a hipótese de que o resíduo atua como um fator estressante no 
cultivo das microalgas. Isso ocorre em virtude do aumento da turbidez do meio, de forma que menos luz penetra no fotobiorreator, o que pode fazer com que a microalga acumule maiores quantidades de lipídios, como afirmado por Hu et al. (2008).

Medidas de Biomassa: Para o acompanhamento da biomassa produzida foram realizadas leituras diárias de absorbância dos fotobiorreatores no comprimento de onda de $570 \mathrm{~nm}$. A curva de calibração utilizada para a conversão dos valores de absorbância para concentração de biomassa foi definida no trabalho de Ramirez (2013). As leituras de absorbância foram realizadas em Espectrofotômetro (UV-1600 Pró-Análise). Para a construção das curvas de crescimento das microalgas os cultivos microalgais foram mantidos nas condições de $20^{\circ} \mathrm{C}, 10000$ lux (intensidade luminosa), fotoperíodo de 12 horas luz e 12 horas escuro e injeção de ar de 25 v.v.m. A relação entre densidade ótica e biomassa, apresentada na Equação 1, foi definida no trabalho de Ramirez (2013).

$$
\text { Biomassa }\left(\frac{g}{L}\right)=0,4669 \times D O_{(570 n m)}-0,0243
$$

Medidas de Espectroscopia de Fluorescência: As leituras de fluorescência foram realizadas em espectrofluorômetro FluoroMax-4, Horiba. As amostras para análise foram preparadas conforme Chen, Sommerfeld et al. (2011), no qual $10 \mu \mathrm{L}$ de cultivo de algas são adicionado à $50 \mu \mathrm{L}$ de DMSO (dimetil sulfóxido) e então colocados em um micro-ondas por 50 segundos em potência alta $(1250 \mathrm{~W})$. Após, são adicionados $0,935 \mathrm{~mL}$ de água destilada e $10 \mu \mathrm{L}$ de solução de Vermelho do Nilo (100 $\mu \mathrm{g} / \mathrm{mL}$ em acetona), sendo novamente colocadas no micro-ondas por mais 60 segundos. A faixa de varredura foi comprimento de onda de excitação entre 220 e $600 \mathrm{~nm}$ e emissão entre $240 \mathrm{~nm}$ e $850 \mathrm{~nm}$ (com incremento de $10 \mathrm{~nm}$ ).

\subsection{Análise de Dados de Espectroscopia de Fluorescência}

Para a extração de informações úteis dos dados espectrais, fez-se o uso da metodologia PSCM (Pure Spectra Chemometric Modeling), associada à ferramenta de otimização heurística "Ant Colony Optimization"- ACO. Esta metodologia permite a obtenção de modelos que utilizam diretamente a intensidade de determinados pares de fluorescência (variáveis independentes) para a predição de estados do sistema (variáveis dependentes). Rotinas e procedimentos de cálculos foram realizados no software MATLAB (Ver. 5.3.0.10183 R11, The Mathworks, Inc., Natick, USA). Devido à pequena quantidade de dados amostrais, optou-se pela utilização da metodologia de "Cross Validation" para teste e comparação de resultados (Li, Morris e Martin, 2002; Ranzan et al., 2014). 


\section{RESULTADOS E DISCUSSÃO}

\subsection{Análise Qualitativa Utilizando-se a Metodologia ACO}

Utilizando-se primeiramente a metodologia ACO, buscaram-se as regiões qualitativamente significativas do plano espectral de fluorescência 2D. Nesta busca foram avaliados modelos multilineares com cinco estruturas diferentes, desde modelos com oito variáveis de entrada (oito pares de fluorescência) até modelos com doze variáveis de entrada distintas (doze pares de fluorescência).

Os modelos gerados com um e dois pares de fluorescência não apresentaram uma segmentação eficiente na distinção de regiões importantes para a referida variável de estado, salientando componentes espectrais isoladamente. Já os modelos gerados com três, quatro e cinco pares apresentam regiões específicas definidas, apresentando um melhor indicativo da assinatura espectral da referida variável. No modelo com quatro pares é apresentada uma possível seleção de regiões significativas, por salientar de forma mais evidente regiões específicas do espectro.

\subsection{Análise Quantitativa de Azeite em Acetona Utilizando EF2D}

A análise PSCM foi realizada em um conjunto de dados composto por quatorze amostras, no qual estas foram analisadas considerando-se apenas a intensidade de fluorescência relativa, ou seja, o espectro de fluorescência da amostra de branco (concentração nula de azeite) foi reduzido das demais amostras.

Os resultados mostram que os modelos que consideram os menores números de variáveis de entrada apresentam os maiores erros (principalmente para as amostras um e oito, que apresentam as menores concentrações de azeite dentro do conjunto de amostras), gerando um problema de escalonamento do gráfico. A Figura 1 apresenta os resultados obtidos na predição da variável de estado, utilizando modelos com maior número de variáveis de entrada (tamanhos de 8 à 12 ou seja, 8 a 12 variáveis de entrada), o erro percentual médio não assume valores superiores à $4 \%$ (nos piores casos), assumindo valores inferiores à $0,5 \%$ para modelos com nove ou mais variáveis de entrada. Os resultados apresentados nesta Figura são referentes à etapa de calibração dos modelos quimiométricos.

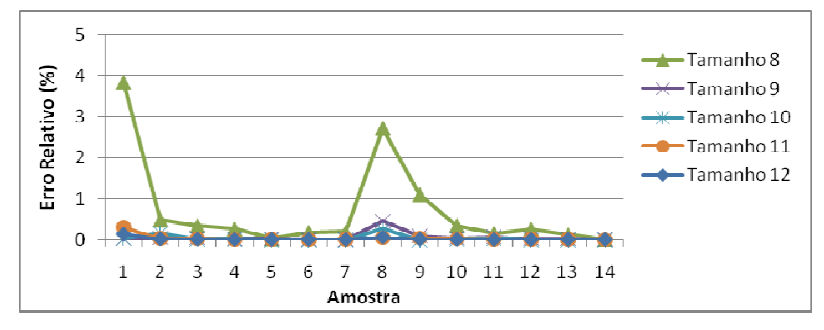

Figura 1: Erro relativo percentual obtido entre a variável predita e a variável medida para catorze amostras de diferentes concentrações de azeite analisadas. 
A ideia principal na seleção de um modelo é a busca pela melhor relação entre robustez (capacidade de predição) e tamanho de modelos (número de variáveis independentes utilizadas para a calibração do modelo). Com base nisso, o modelo selecionado é o de tamanho nove, visto que apresenta margem de erro de medida aceitável aos padrões do experimento (menores que 0,5\%). Estes resultados comprovam e efetividade da metodologia de caracterização proposta, de forma que a quantificação de soluções de azeite em acetona, utilizando o corante Vermelho do Nilo e Espectroscopia Fluorescente 2D, é viável, apresentando erros de predição satisfatórios

\subsection{Cultivo das Microalgas em Vinhaça}

A Figura 2 apresenta as curvas de crescimento de biomassa microalgal no decorrer dos cultivos, em função da concentração de vinhaça presente no meio reacional.

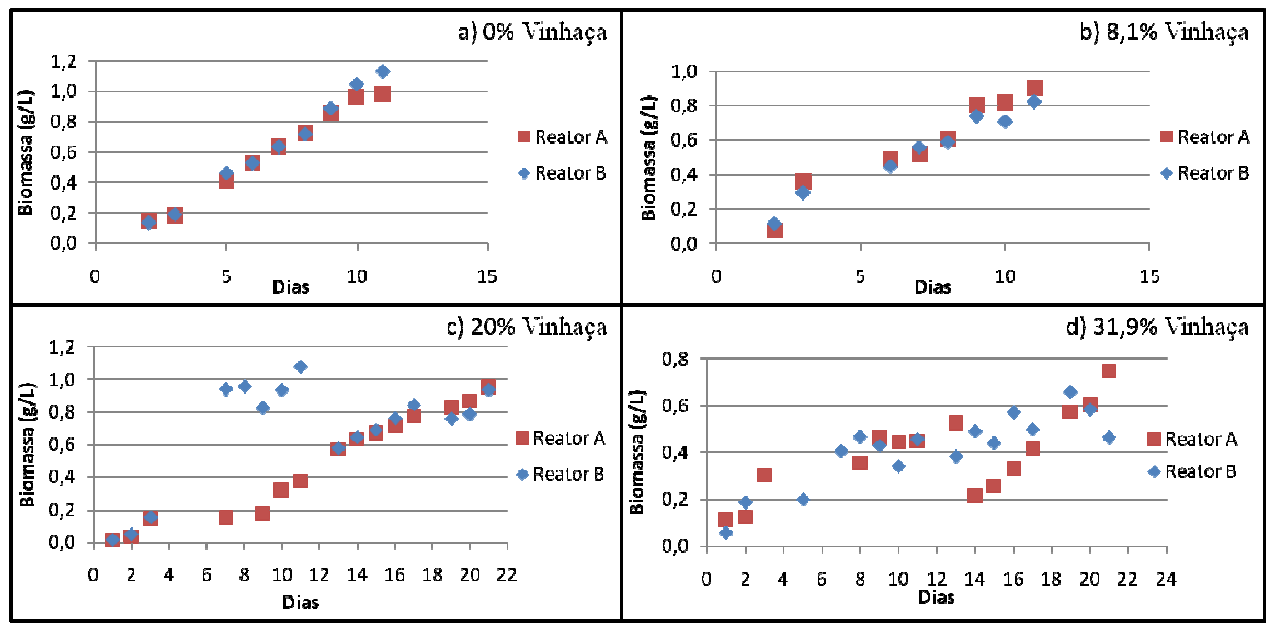

Figura 2: Curvas de crescimento microalgal.

Como pode ser observado nas Figuras 2(a) e Figura 2(b), os fotobiorreatores com 0\% e $8,1 \%$ de vinhaça apresentaram equivalência entre as duplicatas (curva de crescimento Reator A versus curva de crescimento Reator B). Já os reatores com $20 \%$ e 31,9\% (Figura 2(c) e Figura 2(d)), embora apresentem a mesma tendência, limitam-se a apresentar crescimento similar. Isso ocorre porque a vinhaça utilizada continha altos teores de sólidos suspensos que ocasionam diferenças de turbidez entre as duplicatas, fator que influi diretamente no crescimento desses microorganismos e que apresentou a maior evidência nos fotobiorreatores com 31,9\% de vinhaça. Também é observada a ocorrência de floculação dos particulados sólidos, que dificultam a homogeneização dos reatores, sendo que para maiores porcentagens de vinhaça houve maior floculação. Portanto, a discrepância de dados presentes no experimento com $20 \%$ de vinhaça, principalmente entre o quinto e décimo primeiro dias, pode ser devido a erros experimentais ou de caracterização, em decorrência da floculação de sólidos suspensos. 
O cultivo dos fotobiorreatores com $0 \%$ e $8,1 \%$ de vinhaça teve duração de onze dias, como sugerido no trabalho de Ramirez (2013). Entretanto, nos fotobiorreatores com $20 \%$ e $31,9 \%$ de vinhaça, o cultivo foi de vinte e um dias devido a fatores visuais que indicaram um atraso no crescimento das microalgas.

\subsection{Análise de Fluorescência dos Cultivos de Microalgas}

Durante o período de cultivo foram realizadas medidas de espectroscopia fluorescente em intervalos de dois dias visando o acompanhamento do acúmulo lipídico das microalgas. $\mathrm{O}$ objetivo é avaliar a aplicação direta dos modelos desenvolvidos para caracterização lipídica, calibrados com azeite de oliva em acetona, com a finalidade de acompanhar a evolução da concentração de lipídios nos reatores de microalgas, durante os cultivos.

Os resultados da aplicação dos modelos quimiométricos nas amostras de microalgas não foram satisfatórios, uma vez que os modelos não captaram o comportamento dos lipídios nestes micro-organismos. Mesmo para modelos com elevado número de variáveis, tal comportamento se repetiu. A impossibilidade do uso de fluorescência para caracterização de microalgas cultivadas em vinhaça pode ser explicada por alguns fatores, principalmente ligados a este rejeito. Como no começo do cultivo o meio encontrava-se turvo, dificultando a penetrabilidade de luz, as microalgas possivelmente não realizaram fotossíntese e passaram a consumir uma fonte de carbono diferente de $\mathrm{CO} 2$ disponível no meio, o que pode afetar diretamente a forma como o microrganismo produz ou consome lipídios.

Apesar dos resultados insatisfatórios obtidos na avaliação quantitativa da concentração de lipídios em fotobiorreatores, pode ser observado na Figura 3 que os resultados obtidos através das análises de fluorescência seguem a tendência de valores que condiz com o crescimento da biomassa microalgal para os fotobiorreatores com 8,1\% de vinhaça. Este resultado indica que o método foi capaz de captar, mesmo que parcialmente, a variação esperada da variável de estado de interesse. Estes resultados corroboram a viabilidade de acompanhamento do acúmulo lipídico através do método proposto, entretanto, ajustes no método de calibração devem ser propostos de forma a tornar a quantificação da concentração de lipídios viável.

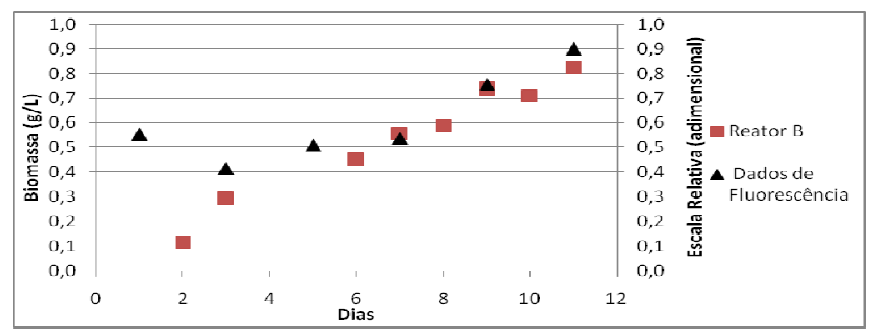

Figura 3: Crescimento da biomassa microalgal (coordenada da esquerda) e variação proporcional, adimensional, do acompanhamento lipídico do cultivo (coordenada da direita) realizado em fotobiorreator $\mathrm{B}$ com $8,1 \%$ de vinhaça,utilizando modelo quimiométrico calibrado com soluções de azeite de oliva e acetona. 


\section{CONCLUSÕES}

O presente trabalho mostrou que a metodologia de quantificação de lipídios estudada não pode ser aplicada diretamente para a quantificação dos lipídios presentes nas microalgas cultivadas em vinhaça. No entanto, os resultados indicam que a metodologia pode ser refinada através de calibrações dos modelos quimiométricos, usando dados e amostras reais do processo, viabilizando o acompanhamento da concentração de lipídios nos fotobiorreatores através de medidas de espectroscopia fluorescente.

A influência do meio reacional na matriz de dados de fluorescência inviabilizou a calibração dos modelos quimiométricos obtidos utilizando amostras de azeite de oliva e acetona, entretanto, a tendência de crescimento microalgal observada para alguns dos reatores confirma a aplicabilidade da técnica proposta.

\section{REFERÊNCIAS}

CHEN, W.; SOMMERFELD, M.; HU, Q. Microwave-assisted Nile red method for in vivo quantification of neutral lipids in microalgae. Biore. Tech., v. 102, n. 1, p. 135-141, 2011.

CHEN, W. et al. A high throughput Nile red method for quantitative measurement of neutral lipids in microalgae. J. of Microbio. Met., v. 77, n. 1, p. 41-47, 2009.

CHISTI, Y. Biodiesel from microalgae beats bioethanol. Trends in Biotech., v. 26, n. 3, p. 126-131, 2008.

CLEMENTSCHITSCH, F. et al. Sensor combination and chemometric modelling for improved process monitoring in recombinant E. coli fed-batch cultivations. J. of Biotech., v. 120, n. 2, p. 183-196, 2005.

GOVENDER, T. et al. BODIPY staining, an alternative to the Nile Red fluorescence method for the evaluation of intracellular lipids in microalgae. Bio. Tech., v. 114, p. 507-511, 2012.

GRIS, G. et al. Laboratory apparatus to evaluate microalgae production. Brazil. Jourl. Chem. Eng. v. 30, p. 487497. Julho 2013.

HU, Q. et al. Microalgaltriacylglycerols as feedstocks for biofuel production: Perspectives and advances. Plant Jourl, v. 54, n. 4, p. 621-639, 2008.

LI, B.; MORRIS, J.; MARTIN, E. B. Model selection for partial least squares regression. Chemo.andIntell. Lab. Syst., v. 64, n. 1, p. 79-89, 10/28/2002.

RAMIREZ, N. N. V. Estudo do Crescimento da Microalga Scenedesmus sp. em Vinhaça. Dissertação de Mestrado - Universidade Federal do Rio Grande do Sul, 2013.

RANZAN, C. et al. Wheat flour characterization using NIR and spectral filter based on ant colony optimization.Chemoms and Intel. Lab. Syst. v. 132, p. 133-140. 15/03/2014. 